Yervant Terzian, Daniel Weedman, Edward Khachikian, eds.

\title{
Feeding the Central Engine in Giant Radio Galaxies
}

\author{
I. F. Mirabel ${ }^{1,2} \&$ O. Laurent ${ }^{1}$ \\ ${ }^{1}$ Service d'Astrophysique. Centre d'Etudes de Saclay. 91191 Gif/Yvette, \\ France. \\ ${ }^{2}$ Instituto de Astronomía y Física del Espacio. c.c. 67, suc 28. (1428) \\ Buenos Aires, Argentina.
}

\begin{abstract}
.
Giant radio galaxies are thought to be massive ellipticals powered by accretion of interstellar matter onto a supermassive black hole. Interactions with gas rich galaxies may provide the interstellar matter to feed the active galactic nucleus (AGN). To power radio lobes that extend up to distances of hundreds of kiloparsecs, gas has to be funneled from kiloparsec size scales down to the AGN at rates of $\sim 1 \mathrm{M}_{\odot} \mathrm{yr}^{-1}$ during $\geq 10^{8}$ years. Therefore, large and massive quasi-stable structures of gas and dust should exist in the deep interior of the giant elliptical hosts of double lobe radio galaxies. Recent mid-infrared observations with ISO revealed for the first time a bisymmetric spiral structure with the dimensions of a small galaxy at the centre of Centaurus A (Mirabel et al. 1999). The spiral was formed out of the tidal debris of accreted gas-rich object(s) and has a dust morphology that is remarkably similar to that found in barred spiral galaxies. The observations of the closest AGN to Earth suggest that the dusty hosts of giant radio galaxies like CenA, are "symbiotic" galaxies composed of a barred spiral inside an elliptical, where the bar serves to funnel gas toward the AGN.
\end{abstract}

\section{A barred spiral at the centre of Centaurus $\mathbf{A}$}

The mid-infrared and submillimeter observations of the dust emission in CenA revealed a bisymmetric structure of $5^{\prime}(\sim 5 \mathrm{kpc}$ for a distance of $3.5 \mathrm{Mpc})$ in total length (Mirabel et al. 1999). Figure 1 shows that in contrast to the optical dark lanes which show a wide and somewhat chaotic distribution, the structure of the mid-infrared emission is remarkably thin, smooth and bisymmetric. The emitting dust is less extended and displaced from the most prominent optical dark lanes. This displacement is not due to major differences between the spatial distributions of the cold and very warm dust components. In a three dimensional tilted and warped disk, projection effects play an important role, and the optical appearance of the dark lanes in a luminous ellipsoidal system may be affected by relatively small amounts of cold dust in the outer parts of the bending disk that are located in the foreground side of the luminous ellipsoidal distribution of stars. 


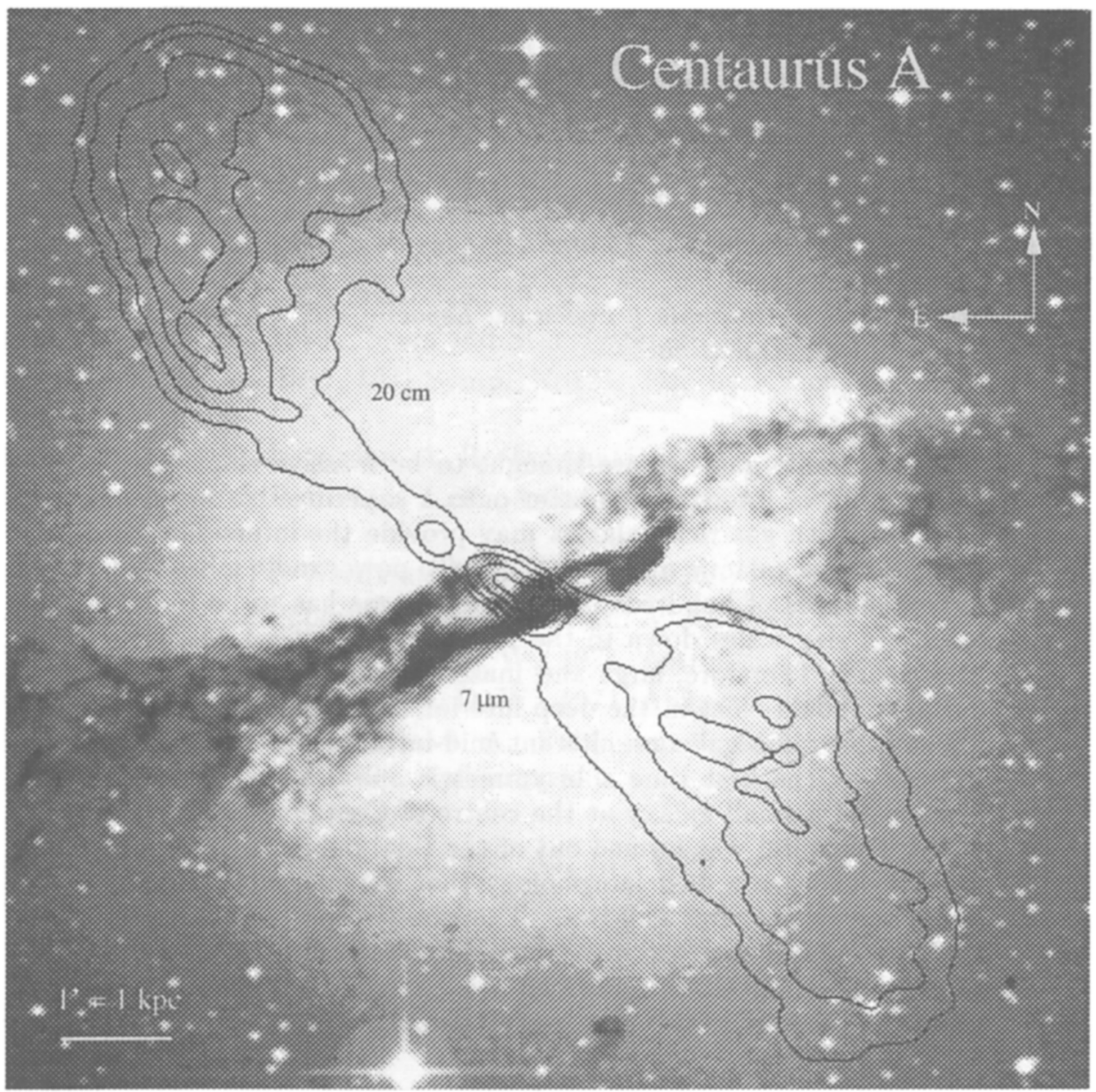

Figure 1. The ISO $7 \mu \mathrm{m}$ emission (dark structure) and VLA $20 \mathrm{~cm}$ continuum in contours (Condon et al. 1996), overlaid on an optical image from the Palomar Digital Sky Survey. The emission from dust with a bisymmetric morphology at the centre is about 10 times smaller than the overall size of the shell structure in the elliptical (Malin et al. 1983) and lies on a plane that is almost parallel to the minor axis of its giant host. Whereas the gas associated to the spiral rotates with a maximum radial velocity of $250 \mathrm{~km} \mathrm{~s}^{-1}$, the ellipsoidal stellar component rotates slowly approximately perpendicular to the dust lane (Wilkinson et al. 1986). The synchrotron radio jets shown in this figure correspond to the inner structure of a double lobe radio source that extends up to $5^{\circ}(\sim 300 \mathrm{kpc})$ on the sky. The jets are believed to be powered by a massive black hole located at the common dynamic center of the elliptical and spiral structures. 
The structures seen in CenA with higher angular resolution by means of the mid-infrared observations with ISO are inside the general distribution of the molecular gas (Eckart et al. 1990; Rydbeck et al. 1993). Figure 2 shows that the interpretation of the bisymmetric structure at the centre of CenA as a barred spiral is fully consistent with the morphology of the dust lanes observed in galaxies classified as barred spirals, and the kinematics observed in CO data. Furthermore, the interpretation of a barred structure is consistent with theoretical models (Athanassoula, 1992) that predict shocks at the leading edge of bars, producing an arc-like appearance of the warm dust. The barred spiral is a dynamic instability, i.e. a density wave in the warped disk of gas and dust.

ISOCAM could not resolve features smaller than $\sim 5^{\prime \prime}$ but $\mathrm{K}(2.2 \mu \mathrm{m})$ band polarization (Packham et al. 1996) due to the passage of starlight through clouds that contain electrons and/or aligned small grains can be used to trace with higher angular resolution the preferential distribution of dust in the innermost central region. The position angle of the polarization in Figure 2 suggests the presence of a secondary bar, or "nuclear" bar of gas of few hundred parsecs in size. This presumed secondary bar inside the primary bar could be the dynamical instability that brings gas towards the supermassive black hole, as proposed in the theoretical model by Shlosman et al. (1989). In fact, molecular gas absorption has been detected in front of the compact nuclear source at millimeter wavelengths, and it has been proposed that the absorption at redshifted velocities represents gas falling into the centre (Israel et al. 1991).

It is believed that the same accretion event(s) that began tearing apart a gas-rich object(s) also created the faint stellar and gaseous shells observed around CenA. It is known that the accretion of a small disk galaxy by a massive elliptical would lead to the complete tidal disruption of the former as it spirals inward in the potential of the elliptical galaxy. In this process the gas decouples from the stars and sinks more readily to the centre, forming a new disk out of the gaseous component alone. In CenA the overall angular momentum of the newly formed disk is not aligned with the major axis of the elliptical. Therefore, the gaseous disk is subject to torques forcing it to warp. Although gas is still settling towards the central regions, the morphological and dynamical symmetry of the spiral indicates that it is a stable structure and not a transient feature. Rotating at $250 \mathrm{~km} \mathrm{~s}^{-1}$, it must have undergone several full rotations depending on how long after the initial encounter it took the gas to settle into the central disk we now see.

Using near infrared photometry as well as the kinematics of the gas, a disk-to-total mass ratio within the turnover radius of the rotation curve of the order of $10^{-2}$ is obtained. N-body simulations would rule out that a low-mass stellar bar has formed spontaneously in the disk, and survived at steady-state. However these simulations consider stellar disks, while here we are dealing with a gaseous one. Using the much lower velocity dispersion (typically $5-10 \mathrm{~km} \mathrm{~s}^{-1}$ in the gas, compared with $50 \mathrm{~km} \mathrm{~s}^{-1}$ for a stellar disk and $145 \mathrm{~km} \mathrm{~s}^{-1}$ for the spheroidal component in CenA (Wilkinson et al. 1986, Eckart et al. 1990) a much lower Toomre's Q parameter (of the order of 1 ) is derived for the gaseous disk in CenA. Therefore, a gaseous disk is much more self-gravitating than a stellar one of similar mass, and the bar in CenA can be in a quasi-steady state and might have formed spontaneously, or be driven. 


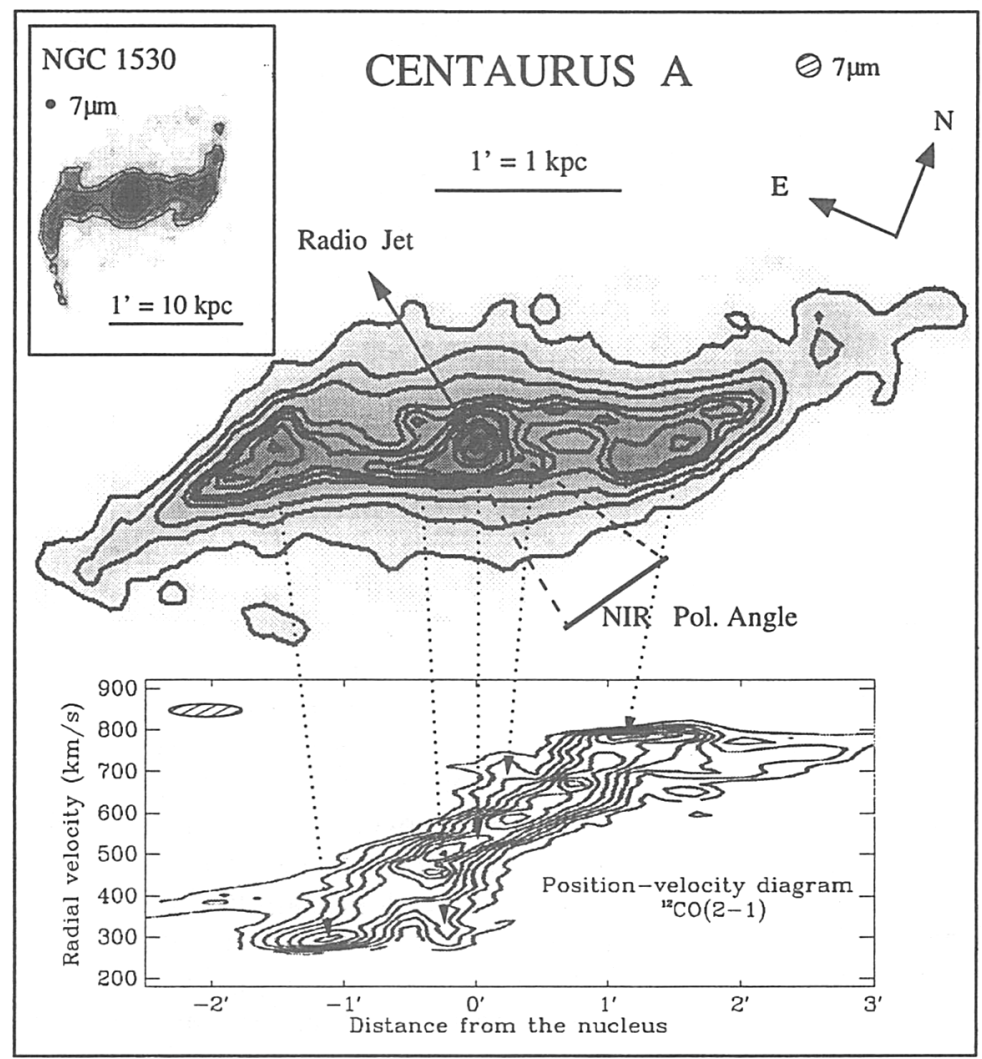

Figure 2. ISO $7 \mu \mathrm{m}$ image and ${ }^{12} \mathrm{CO}(2-1)$ position-velocity map (Quillen et al. 1992) of the central region of CenA. Note the similarity in morphology with the $7 \mu \mathrm{m}$ image of the prototype barred spiral NGC 1530 . In NGC 1530 the plane that contains the bar is tilted by $\sim 55^{\circ}$ to the line of sight, whereas in CenA it is tilted by $\sim 18^{\circ}$. The overall structure exhibited by the $7 \mu \mathrm{m}$ emission from CenA is that of a barred spiral with a primary bar extending $\sim 1^{\prime}$ in radius from the nucleus, connected in its outer ends to trailing spiral arms. The strongest $7 \mu \mathrm{m}$ emission from the primary bar is along its leading edge in what takes the form of two slightly curved arcs, where shocks and density enhancements take place. The inner ends of these two arcs are connected to what may be a secondary nuclear bar whose position angle is defined by the NIR $\mathrm{K}(2.2 \mu \mathrm{m})$ band polarization (Packham et al. 1996). On the plane of the sky the radio jets appear perpendicular to the innermost polarization angle. The kinematics of the gas in the lower panel is consistent with a barred spiral, where the bar rotates as a rigid body within $70^{\prime \prime}$, whereas at radii larger than $\pm 70^{\prime \prime}$ the gas exhibits the differential rotation (flat rotation curve) typical of galactic disks. 


\section{The symbiotic galaxy Centaurus A: a template for giant radio galaxies}

Heckman et al. (1986) have shown that radio galaxies with giant radio lobes have peculiar optical morphologies. In this respect, CenA is not an exception and could serve as a well-positioned template to examine in detail the clues to the origin and evolution of activity in early-type radio galaxies with the same radio morphology, namely, with giant double radio lobes. Prominent dust bands are frequently observed in the hosts of this type of radio galaxy. Fornax A, the second nearest giant radio galaxy after Cen A, exhibits clear signs for the merger of gas-rich galaxies with a dusty early type galaxy. Cygnus $\mathrm{A}$, the prototype radio galaxy with double morphology, is crossed by prominent optically dark bands that contain $\sim 10^{8} \mathrm{M}_{\odot}$ of dust (Robson et al. 1998). It has been shown that in dusty radio galaxies with double radio structure, the dust is usually found perpendicular to the radio axis (Kotani \& Ekers 1979; van Dokkum \& Franx 1995) which suggests a connection between the mechanism leading to these double radio morphologies and the rotation axis of the dusty disk.

The specific mechanism in rapidly rotating disks of gas and dust that brings fuel to the central engine in radio loud AGNs has been difficult to probe observationally for several reasons. First, galaxies like CenA are at greater distances (for instance, Fornax A is 5-10 times and Cygnus A is $\sim 70$ times more distant than CenA), and at those distances it is difficult to see the detailed morphology of dust and gas on scales $\leq 100 \mathrm{pc}$. Second, at optical and near-infrared wavelengths the light from the old stellar population with a giant ellipsoidal distribution overwhelms any emission from dust and newly formed stars in the deep interior. It is in the mid-infrared that the emission from very warm dust can be better traced, and to this end we had to wait for the unprecedented capabilities of ISOCAM. Third, the observation of the cold gas distribution by means of millimeter observations of weak molecular line emission on top of the strong continuum of powerful radio galaxies is a difficult task.

The need for the presence of bars in AGNs has been questioned because the ocurrence of bars in Seyfert galaxies is not higher than in normal galaxies (McLeod \& Riecke, 1995; Ho et al. 1997; Mulchaey \& Regan, 1997). Furthermore, using HST images in the optical and near-infrared, no signatures of dust absorption with barred structures have been found in Seyfert 2's (Regan \& Mulchaey, 1999). However, it is difficult to trace the presence of dust inside bulge and ellipsoidal systems using only optical and near-infrared observations because at those wavelengths the stellar emission dominates. Mirabel et al. (1999) have shown that the true structure of the dust in the deep interior of CenA can only be revealed by the emission at wavelengths longer than $3 \mu \mathrm{m}$. In CenA the dust absorption derived from colors between the near-infrared and the optical (Quillen, 1993) only trace the foreground side of the structure shown in Figures 1 and 2.

It is believed that the AGN activity in Seyfert galaxies is much more sporadic than in giant radio galaxies and that Seyferts may require accretion masses with sizes that are orders of magnitude smaller than those needed to power the giant lobes of radio galaxies. The observation of emission from the accreting fuel in the central regions of Seyfert galaxies may have to await the high angular resolution and sensitivity of the NGST and MMA/LSA. 
The barred spiral at the centre of CenA has dimensions comparable to that of the small Local Group galaxy Messier 33. It lies on a plane that is almost parallel to the minor axis of the giant elliptical. Whereas the spiral rotates with maximum radial velocities of $\sim 250 \mathrm{~km} \mathrm{~s}^{-1}$, the ellipsoidal stellar component seems to rotate slowly (maximum line-of-sight velocity is $\sim 40 \mathrm{~km}$ $\mathrm{s}^{-1}$ ) approximately perpendicular to the dust lane. The genesis, morphology, and dynamics of the spiral formed at the centre of CenA are determined by the gravitational potential of the elliptical, much as a usual spiral with its dark matter halo. On the other hand, the AGN that powers the radio jets is fed by gas funneled to the center via the bar structure of the spiral. The spatial co-existence and intimate association between these two distinct and dissimilar systems suggest a "symbiotic" association.

\section{Conclusions}

1) The observation of dust emission from the centre of CenA opens the more general question on whether the hosts of giant radio galaxies are symbiotic galaxies composed of spirals at the centre of giant ellipticals.

2) The true structure of dust in the deep interior of ellipsoidal stellar systems is better traced by emission from dust at wavelengths longer than 3 microns, rather than by absorption using colors from near-infrared and optical photometry.

3) The accreting matter in Seyferts is likely to have masses and sizes that are several orders of magnitude smaller than in giant radio galaxies. To trace the accreting gas and dust in Seyferts, the angular resolution and sensitivity of the NGST and MMA/LSA are needed.

\section{References}

Athanassoula E., 1992, MNRAS, 259, 345

Condon J.J., Helou G., Sanders D.B., Soifer B.T., 1996, A\&AS, 103, 81

Eckart A., Cameron M., Genzel R. et al., 1990a, ApJ, 365, 522

Heckman, T.M. et al., 1986, ApJ, 311, 526

Ho L., Filippenko A. \& Sargent W., 1997, ApJ, 487, 591

Israel F.P., van Dishoeck E.F., Baas F. et al., 1991, A\&A, 245, L13

Kotanyi C.G., Ekers R.D., 1979, A\&A, 73, L1

Malin D.F., Quinn P.J., Graham J.A., 1983, ApJ, 272, L5

McLeod K.K. \& Rieke G.H. 1995, ApJ, 441, 96

Mirabel I.F., Laurent O., Sanders D.B. et al. 1999, A\&A, 341, 667

Mulchaey J.S. \& Regan M.W., 1997, ApJ, 482, L135

Packham C., Hough J.H., Young S. et al., 1996, MNRAS, 278, 406

Quillen A.C., de Zeeuw P.T., Phinney E.S., Phillips T.G., 1992, ApJ, 391, 121

Quillen A.C., Graham J.R., Frogel J.A., 1993, ApJ, 412, 550

Regan M.W. \& Mulchaey J.S. 1999, AJ, in press

Robson E.I., Leeuw L.L., Stevens J.A., Holland W.S., 1998, MNRAS, 301, 935 
Rydbeck G., Wiklind T., Cameron M. et al., 1993, A\&A, 270, L13

Shlosman I., Frank J., Begelman M.C., 1989, Nature, 338, 45

van Dokkum P.G., Franx M., 1995, AJ110, 2027

Wilkinson A., Sharples R.M., Fosbury R.A., Wallace P.T., 1986, MNRAS, 218, 297

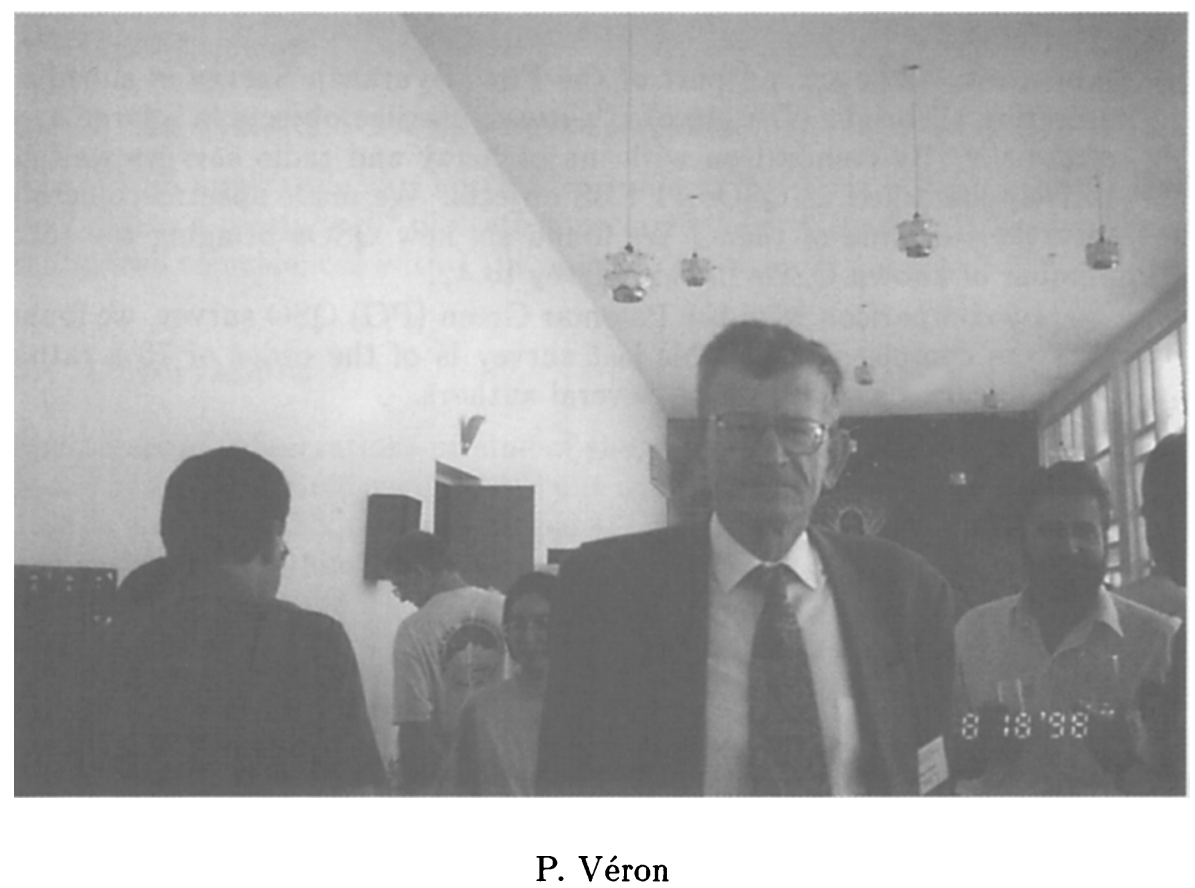

